\title{
Note on Mr Tweedie's Theorem in Geometry.
}

By Peter Pinkerton, M.A.

Let $\mathrm{ABC}, \mathrm{A}^{\prime} \mathrm{B}^{\prime} \mathrm{C}^{\prime}$ (Fig. 4) be two triangles equiangular in the same sense. Let $B C, B^{\prime} C^{\prime}$ meet in $X$. Describe circles round $\mathrm{BXB}^{\prime}, \mathrm{CXC}^{\prime}$ to meet again in $\mathrm{O}$. Then it is easy to see that the triangles $\mathrm{BOC}, \mathrm{COA}, \mathrm{AOB}$ are equiangular in the same sense to the triangles $\mathrm{B}^{\prime} O \mathrm{C}^{\prime}, \mathrm{C}^{\prime} O \mathrm{~A}^{\prime}, \mathrm{A}^{\prime} O \mathrm{~B}^{\prime}$ respectively. Hence the triangles $\mathrm{AOA}^{\prime}, \mathrm{BOB}^{\prime}, \mathrm{COC}^{\prime}$ are similar ;

$$
\therefore \quad \frac{\mathrm{AA}^{\prime}}{\mathrm{AO}}=\frac{\mathrm{BB}^{\prime}}{\mathrm{BO}}=\frac{\mathrm{CC}^{\prime}}{\mathrm{CO}}
$$

$\therefore a . \mathrm{AA}^{\prime}, b . \mathrm{BB}^{\prime}, c . \mathrm{CC}^{\prime}$ are proportional to $a . \mathrm{AO}, b . \mathrm{BO}, c . \mathrm{CO}$, where $a, b, c$ are the sides of the triangle $\mathrm{ABC}$.

From $O$ draw $O P, O Q$, OR perpendicular to $B C, C A, A B$ respectively.

$$
\text { Then } \begin{aligned}
\mathrm{QR} & =\mathrm{AO} \sin \mathrm{A} \propto a . \mathrm{AO}, \\
\mathrm{RP} & =\mathrm{BO} \sin \mathrm{B} \propto b . \mathrm{BO}, \\
\mathrm{PQ} & =\mathrm{CO} \sin \mathrm{C} \propto c . \mathrm{CO} ;
\end{aligned}
$$

$\therefore a . \mathrm{AA}^{\prime}, b . \mathrm{BB}^{\prime}, c . \mathrm{CC}^{\prime}$, being proportional to $a . \mathrm{AO}, b . \mathrm{BO}, c . \mathrm{CO}$, are proportional to $Q R, R P, P Q$.

But $P Q R$ is a triangle, unless $O$ is on the circumcircle of $A B C$ when $P Q R$ is the Simson line of $O$.

$\therefore \mathrm{QR}+\mathrm{RP}>\mathrm{PQ}$, with two similar inequalities, except that one of the inequalities becomes an equality if $O$ is on the circumcircle of ABC.

$\therefore a \cdot \mathrm{AA}^{\prime}+b \cdot \mathrm{BB}^{\prime}>c . \mathrm{CC}^{\prime}$, with two sinilar inequalities; one of the inequalities becoming an equality when $O$ lies on the circumcircle of $\mathrm{ABC}$.

Similarily in the case of an equality $O$ lies also on the circumcircle of $\mathbf{A}^{\prime} \mathbf{B}^{\prime} \mathbf{C}^{\prime}$.

For the case of equilateral triangles $a=b=c$;

$\therefore \mathrm{AA}^{\prime}+\mathrm{BB}^{\prime}>\mathrm{CC}^{\prime}$, with two similar inequalities; one of the three inequalities becoming an equality when $O$ lies on the circumcircles of $A B C$ and $A^{\prime} B^{\prime} C^{\prime}$.

It is obvious that the theorem reduces to Ptolemy's Theorem or its converse. 